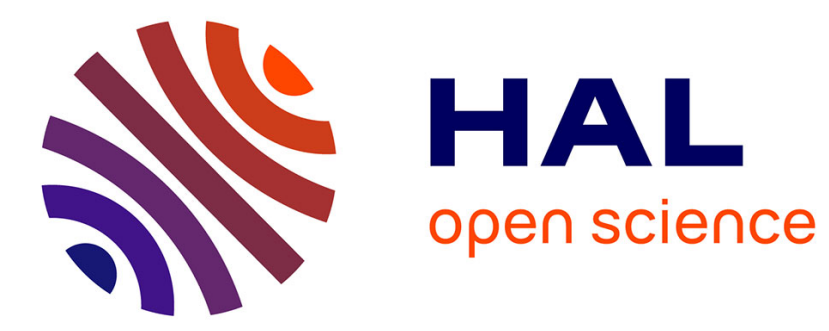

\title{
One-component plasma in a strong uniform magnetic field
}

B. Bernu

\section{To cite this version:}

B. Bernu. One-component plasma in a strong uniform magnetic field. Journal de Physique Lettres, 1981, 42 (12), pp.253-255. 10.1051/jphyslet:019810042012025300 . jpa-00231921

\section{HAL Id: jpa-00231921 https://hal.science/jpa-00231921}

Submitted on 1 Jan 1981

HAL is a multi-disciplinary open access archive for the deposit and dissemination of scientific research documents, whether they are published or not. The documents may come from teaching and research institutions in France or abroad, or from public or private research centers.
L'archive ouverte pluridisciplinaire HAL, est destinée au dépôt et à la diffusion de documents scientifiques de niveau recherche, publiés ou non, émanant des établissements d'enseignement et de recherche français ou étrangers, des laboratoires publics ou privés. 


\title{
One-component plasma in a strong uniform magnetic field (*)
}

\author{
B. Bernu \\ Laboratoire de Physique Théorique des Liquides (**), Université Pierre et Marie Curie, \\ 4, place Jussieu, 75230 Paris Cedex 05, France
}

(Reçu le 3 mars 1981, accepté le 29 avril 1981)

Résumé. - A partir d'expériences de Dynamique Moléculaire pour le plasma à un composant en champ magnétique intense et uniforme, nous avons calculé les fonctions de corrélation des vitesses et les coefficients de selfdiffusion. En couplage fort, des résultats inattendus sont observés.

Abstract. - We present Molecular Dynamics computations of the velocity autocorrelation function and the self-diffusion coefficients of the one-component plasma in a strong and uniform constant magnetic field.

The one-component plasma (OCP) is a simple model for fully ionized matter made up of classical point ions moving in a uniform and rigid background of degenerate electrons $\left(T \ll T_{\mathrm{F}}\right.$, where $T_{\mathrm{F}}$ is the Fermi temperature of the electron gas).

The reduced static properties of the OCP depend on the single dimensionless coupling parameter $\Gamma=(Z e)^{2} /\left(a k_{\mathrm{B}} T\right)$, where $Z$ is the ionic charge, $a=(3 / 4 \pi \rho)^{1 / 3}$ is the "ion sphere " radius and $\rho=N / V$ is the number density. The natural time scale to describe the dynamical properties of the model is the inverse of the plasma frequency

$$
\omega_{\mathrm{p}}=\left(4 \pi \rho(Z e)^{2} / m\right)^{1 / 2},
$$

where $m$ is the ion mass.

We have studied the influence of a magnetic field on the velocity autocorrelation function (v.a.f.). The magnetic field is sufficiently strong that the magnetic forces are of the same order of magnitude as the Coulomb forces, i.e. the frequencies $\omega_{\mathbf{B}}\left({ }^{1}\right)$ and $\omega_{\mathrm{p}}$ are comparable. The corresponding magnetic field is several million tesla, which occurs in some stars. The ions will be always classical if their kinetic energy is greater than $h \omega_{\mathrm{B}}$, i.e. for us when $h \omega_{\mathrm{p}} \ll k_{\mathrm{B}} T$.

The linear self-diffusion coefficients can be expressed

(*) This work was supported in part by the Commissariat à l'Energie Atomique under the contract number DAM/CEL 903/S.

(**) Equipe associée au C.N.R.S.

(') $\omega_{\mathrm{B}}$ is the Larmor frequency defined by $\omega_{\mathrm{B}}=Z e B / m c$, where $B$ is the magnetic field. in Statistical Mechanics by the usual Kubo formulae :

where

$$
D_{\alpha}=\frac{k_{\mathrm{B}} T}{m} \int_{0}^{\infty} d t Z_{\alpha}(t),
$$

$$
Z_{\alpha}(t)=\frac{m}{k_{\mathrm{B}} T}\left\langle V_{i \alpha}(t) V_{i \alpha}(0)\right\rangle
$$

is the normalized v.a.f. and $\alpha$ designates the $x, y$ or $z$ axis direction. In the following, we put the magnetic field in the $z$ direction.

First we give an estimate of the coefficients $D_{x}$ and $D_{z}$, by taking for the memory function of $Z(t)$ a gaussian form determined by the exact short time behaviour of $Z(t)$ :

$$
\begin{aligned}
& Z_{z}(t)=1-\omega_{\mathrm{p}}^{2} \frac{t^{2}}{2 !} \frac{1}{3}+\omega_{\mathrm{p}}^{4} \frac{t^{4}}{4 !} \times \\
& \times\left(\frac{4}{3} I_{-4}+\frac{1}{9}+K\right)+\cdots[1] \\
& Z_{x}(t)=Z_{z}(t)-\omega_{\mathrm{p}}^{2} \frac{t^{2}}{2 !} b^{2}+\omega_{\mathrm{p}}^{4} \frac{t^{4}}{4 !} b^{2}\left(b^{2}+1\right)+\cdots
\end{aligned}
$$

where

$$
b=\omega_{\mathrm{B}} / \omega_{\mathrm{p}}, I_{-4}=\int_{0}^{\infty} \mathrm{d} x \frac{g_{2}(x)}{x^{4}}, \text { with } x=r / a,
$$

and

$$
\begin{aligned}
K=\frac{1}{2} \int_{0}^{\infty} & \frac{\mathrm{d} x}{x} \int_{0}^{\infty} \frac{\mathrm{d} x^{\prime}}{x^{\prime}} \times \\
& \times \int_{-1}^{+1} \mathrm{~d} \cos \theta\left[g_{3}\left(x, x^{\prime}, \cos \theta\right)-g_{2}(x) g_{2}\left(x^{\prime}\right)\right] \\
& \times\left(3 \cos ^{2} \theta-1\right)
\end{aligned}
$$


with $g_{2}(x)$ and $g_{3}\left(x, x^{\prime}, \cos \theta\right)$ the two-body and threebody static correlation function. From these equations, we deduce the following form of the self-diffusion coefficients :

$$
\begin{aligned}
& D_{z}(b)=D_{z}(0)=\frac{k_{\mathrm{B}} T}{m} \frac{3}{\omega_{\mathrm{p}}}\left(\frac{8 I_{-4}+6 K}{\pi}\right)^{1 / 2} \\
& D_{x}(b)=\frac{k_{\mathrm{B}} T}{m} \frac{3}{\omega_{\mathrm{p}}}\left(\frac{8 I_{-4}+6 K+9 b^{2}}{\pi\left(1+3 b^{2}\right)^{3}}\right)^{1 / 2} .
\end{aligned}
$$

Note that $D_{z}(b)$ is independent of the magnetic field.

In this note, we present numerical estimates of the self-diffusion coefficients, obtained for the strongly coupled $(\Gamma \simeq 1,10$ and 100$)$ OCP by molecular dynamics (MD) computer simulations. The simulations have been performed for periodic systems of 128 (at $\Gamma \simeq 1)$ and $250(\Gamma \simeq 10$ and 100) particles [2]. The total time intervals $\omega_{\mathrm{p}} T$ over which the equations of motion were integrated and the numerical values of the self-diffusion coefficients are given in table I. The statistical uncertainties of the amplitudes are of the order of $2 \%$. After a time $t \simeq 40 \omega_{\mathrm{p}}^{-1}$, the amplitudes drop below the noise level.

Table I. $-T:$ Total integration time of the equations of motion in units of $\omega_{\mathrm{p}}^{-1} \cdot b:$ Magnetic field in units of $\omega_{\mathrm{p}} \frac{m c}{Z e} . D^{*}:$ Self-diffusion coefficients in units of $\omega_{\mathrm{p}} a^{2}$, with $x$ (resp. z) for orthogonal (resp. parallel) to the

\begin{tabular}{|c|c|c|c|c|c|c|}
\hline$\Gamma$ & $\omega_{\mathrm{p}} T$ & $b$ & $D_{x}^{*}$ & $D_{z}^{*}$ & $D_{x}^{*}$ (Eq. (5)) & $D_{z}^{*}$ (Eq. (4)) \\
\hline$\overline{1}$ & 216 & $\overline{01}$ & $\overline{48}$ & $\overline{90}$ & - & $3 \overline{5}$ \\
\hline & 120 & 1.0 & 0.063 & 1.90 & 0.45 & 3.5 \\
\hline 9.7 & $\begin{array}{l}140 \\
120\end{array}$ & $\begin{array}{l}0.3 \\
1.0\end{array}$ & $\begin{array}{l}0.073 \\
0.029\end{array}$ & $\begin{array}{l}0.13 \\
0.085\end{array}$ & $\begin{array}{l}0.055 \\
0.014\end{array}$ & $\begin{array}{l}0.078 \\
0.078\end{array}$ \\
\hline & $\begin{array}{l}144 \\
288\end{array}$ & $\begin{array}{l}0.3 \\
1.0\end{array}$ & $\begin{array}{l}0.0046 \\
0.0023\end{array}$ & $\begin{array}{l}0.0060 \\
0.0024\end{array}$ & $\begin{array}{l}0.0039 \\
0.0012\end{array}$ & $\begin{array}{l}0.006 \\
0.006\end{array}$ \\
\hline
\end{tabular}
magnetic field.

From the v.a.f. we have computed their time Laplace transform (noted $\tilde{Z}(\omega)$ ). Then the coefficients $D_{\alpha}$ can be expressed by

$$
D_{\alpha}=\frac{k_{\mathrm{B}} T}{m} \tilde{Z}_{\alpha}(0)
$$

In figures 1 and 2, we give the spectra at $\Gamma=10$ and 100 for the magnetic fields $\omega_{\mathrm{B}}=0.3 \omega_{\mathrm{p}}$ and $\omega_{\mathbf{B}}=1.0 \omega_{\mathrm{p}}$ and without magnetic field (from Ref. [2]).

At $\Gamma=1$, some computations have been performed for magnetic field between $\omega_{\mathrm{B}}=0.0$ and $\omega_{\mathrm{B}}=0.55 \omega_{\mathrm{p}}$ by E. L. Pollock [3] and for $\omega_{\mathrm{B}}=0.1$ and $\omega_{\mathrm{B}}=1.0 \omega_{\mathrm{p}}$ by the author. The results are remarkably simple : the functions $Z_{z}(t)$ are not perturbed by the magnetic field according to (2) and (4), and the functions $Z_{x}\left(t, \omega_{\mathrm{B}}\right)$ can be calculated from $Z_{z}(t)$, with very good precision, by multiplying them by $\cos \omega_{\mathrm{B}} t$. Because $\cos \omega_{\mathrm{B}} t$ represent the v.a.f. of charges in a magnetic

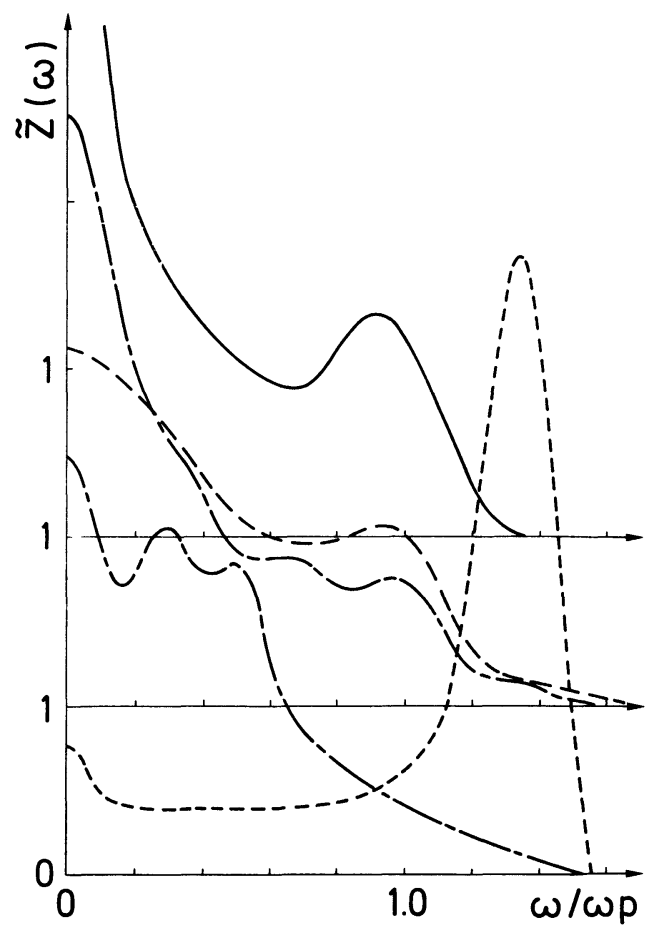

Fig. 1. - v.a.f. spectra at $\Gamma=9.7:-$ without magnetic field [2], $\ldots . . \tilde{Z}_{x}^{\prime}(\omega), \ldots-\square-\tilde{Z}_{z}^{\prime}(\omega)$ for $\omega_{\mathrm{B}}=0.3 \omega_{\mathrm{p}}$ middle curve and $\omega_{\mathrm{B}}=1.0 \omega_{\mathrm{p}}$, bottom curve.

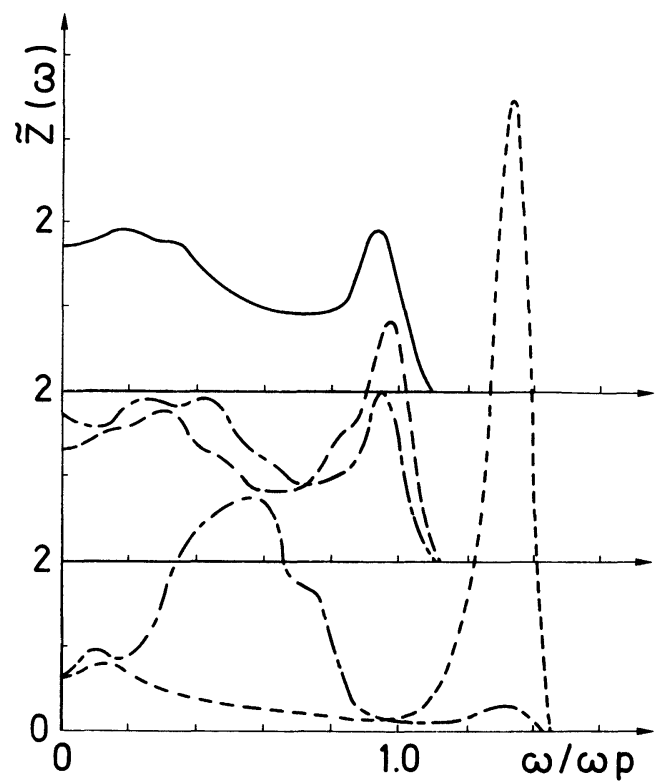

Fig. 2. - Same as figure 1 at $\Gamma=95$, except that - is for $\Gamma=110.4$ [2].

field without coulombic interaction, the physical significance of these results is the disconnecting of the electrostatic and magnetic effects. This is explained by the collision number required to modify the velocity direction of one particle. Consequently we find very simple empirical formulae for the self-diffusion coefficients :

$D_{z}\left(\omega_{\mathrm{B}}\right)=\frac{k_{\mathrm{B}} T}{m} \tilde{Z}^{\prime}(0) \quad$ and $\quad D_{x}\left(\omega_{\mathrm{B}}\right)=\frac{k_{\mathrm{B}} T}{m} \tilde{Z}^{\prime}\left(\omega_{\mathrm{B}}\right)$, 
where $\tilde{Z}^{\prime}(\omega)$ denotes the real part of $\tilde{Z}(\omega)$ without the magnetic field.

At $\Gamma=10$ and 100 , the situation is completely different. The comparison of the spectra in "strong" magnetic field $\left(\omega_{\mathrm{B}}=1.0 \omega_{\mathrm{p}}\right)$ with the ones without magnetic field suggest three important remarks :

i) Transfer of the "plasmon peak " to the high frequencies $\left(\omega=1.3 \omega_{\mathrm{p}}\right.$ instead $0.91 \omega_{\mathrm{p}}$ [2]), and very important increase of the height of this peak for the $\tilde{Z}_{x}(\dot{\omega})$ function.

ii) Total disappearance of the " plasmon peak " for the $\tilde{Z}_{z}(\omega)$ function.

iii) The effects of the magnetic field are qualitatively unlike the ones obtained in weak coupling $(\Gamma=1)$.

With weaker magnetic field $\left(\omega_{\mathrm{B}}=0.3 \omega_{\mathrm{p}}\right)$, the spectra are intermediate between those without field and those with strong magnetic field.

We can understand the points i) and iii) as following :

i) The increasing of the oscillation frequency of $Z_{x}(t)$ owing to the magnetic field is explained by a simple model of a harmonic charged oscillator in a magnetic field. When $\omega_{\mathrm{B}}=\omega_{\mathrm{p}}$ and the eigen frequency $\omega_{0}=0.91 \omega_{\mathrm{p}}$, we find $1.6 \omega_{\mathrm{p}}$ for the resonant frequency at $\Gamma=10$ and 100 . For the other studied values of the coupling and the magnetic field, this model takes account qualitatively of the magnetic field effects on $Z_{x}(t)$.

iii) But with a one-body model, the magnetic field does not modify $Z_{z}(t)$. We have to introduce the colli- sions. Now, in contrast to the weak coupling case, a single collision may be sufficient to change the velocity direction of a particle : when a velocity is parallel to the field B, it was orthogonal to $B$ just before, and consequently it remembers the presence of the magnetic field. This explains the big influence of the magnetic field on $Z_{z}(t)$ in strong coupling and not in weak coupling.

ii) No explanation is found to take account of the complete disappearance of the "plasmon peak " of $Z_{z}(t)$ in strong coupling and strong magnetic field.

By comparing the self-diffusion coefficients obtained from equations (4-5) and those obtained from simulation data (see table I), we establish that the order of magnitude is reasonable, but the predictions of $D_{x}\left(\omega_{\mathrm{B}}\right)$ become worse when the coupling becomes weak and the magnetic field strong. Above all $D_{z}$ does not depend on $\omega_{\mathrm{B}}$. The usual models, from the exact short time expansion of $Z(t)$ cannot take account of the variation of $D_{z}$ with the magnetic field. Finally, we find that in all the cases studied, the magnetic field effect is to decrease the self-diffusion coefficients. The effect is stronger for intense magnetic fields.

Acknowledgments. - The author thanks E. L. Pollock for providing him with unpublished material and acknowledges useful discussions with J. P. Hansen, J. M. Caillol and P. Vieillefosse and is indebted to M. Nelkin for a careful reading of the manuscript.

\section{References}

[1] Vieillefosse, P., Thèse de Doctorat de 3 e Cycle de l'Université Pierre et Marie Curie. Hansen, J. P. : in Strongly Coupled Plasmas, ed. by G. Kalman and P. Carini, (Plenum Pub. Co.. New York) 1978. p. 117.
[2] Hansen, J. P., Mac Donald, I. R. and Pollock, E. L., Phys. Rev. A 11 (1975) 1025.

[3] Pollock, E. L., private communication. 